\title{
Neuromyelitis optica presenting with relapses under treatment with natalizumab: a case report
}

\author{
De-Hyung Lee ${ }^{1 *}$, Alexandra B Laemmer ${ }^{1}$, Anne Waschbisch', Tobias Struffert², Christian Maihöfner ${ }^{1}$, \\ Stefan Schwab ${ }^{1}$ and Ralf Andreas Linker ${ }^{1}$
}

\begin{abstract}
Introduction: Neuromyelitis optica is an inflammatory demyelinating disease of the central nervous system. To date, optimal therapeutic approaches for neuromyelitis optica have yet to be defined. Natalizumab is highly effective in relapsing-remitting multiple sclerosis and might be considered as an option.
\end{abstract}

Case presentation: Here, we describe a 67-year-old Caucasian man with definite neuromyelitis optica with detection of anti-aquaporin-4 antibodies over the course of the disease. After initially discussing the diagnosis of multiple sclerosis at an outside hospital, our patient received interferon beta 1a as well as repeated corticosteroid pulses without success. Under subsequent therapy with natalizumab, he continued to present relapses. It was not until discontinuation of natalizumab, repeated cycles of plasma exchanges and initiation of therapy with rituxan that the disease course started to stabilize. Although B cells were completely depleted, our patient experienced another severe myelitis relapse during further follow-up and an additional immunosuppressive therapy with cyclophosphamide was started. Under this regimen, no further relapses occurred over the next 24 months.

Conclusions: This case adds further evidence to the previously discussed notion that natalizumab, while highly effective in multiple sclerosis, may not work sufficiently in neuromyelitis optica. It further advocates for repetitive testing of anti-aquaporin-4 antibodies before and after treatment initiation.

Keywords: Neuromyelitis optica, Natalizumab, Multiple sclerosis, Rituxan, Anti-AQP-4 antibodies, Seroconversion

\section{Introduction}

Neuromyelitis optica (NMO) is an immune-mediated demyelinating disease with predominant involvement of the optical nerve and the spinal cord. In contrast to multiple sclerosis (MS), NMO is associated with autoantibodies that target the aquaporin-4 water channel on astrocytes (anti-AQP-4 antibody). Anti-AQP-4 antibodies have both high specificity and sensitivity for NMO. AQP-4 seropositivity has been incorporated as additional criterion for the diagnosis of NMO [1,2]. For the treatment of NMO, small trials hint at positive effects of azathioprine, mitoxantrone, mycophenolate motefil and intravenous immunoglobulins, while several observations support the opinion that beta interferons have no effects or may even be harmful in NMO patients $[3,4]$. Nowadays, monoclonal antibodies targeting B cells such as rituxan play an increasingly important role

\footnotetext{
* Correspondence: De-Hyung.Lee@uk-erlangen.de

${ }^{1}$ Department of Neurology, Friedrich-Alexander University of

Erlangen-Nuremberg, Schwabachanlage 6, 91054 Erlangen, Germany

Full list of author information is available at the end of the article
}

in NMO therapy [5]. In addition, effects of further monoclonals like tocilizumab, eculizumab or natalizumab may be discussed [6,7].

\section{Case presentation}

Without significant previous medical history, a 67-year-old Caucasian man developed spinal symptoms with temporary hypesthesia and hypoalgesia in both legs. These symptoms spontaneously resolved without any specific diagnosis at that time. At the age of 73, our patient suffered from bilateral optic neuritis and he was diagnosed with MS at an outside hospital. His expanded disability status scale (EDSS) score was at that time 2.5. Magnetic resonance imaging (MRI) studies of the spinal cord revealed a diffuse cord swelling and longitudinally extensive T2 hypertensive lesions extending from $\mathrm{C} 2$ to $\mathrm{T} 3$ (see Figure 1 depicting a T2-weighted MRI scan, which shows residual longitudinal myelitis with extensive cord atrophy). A cranial MRI scan displayed few periventricular and cerebellar lesions without contrast enhancement and without fulfilling the Barkhof 


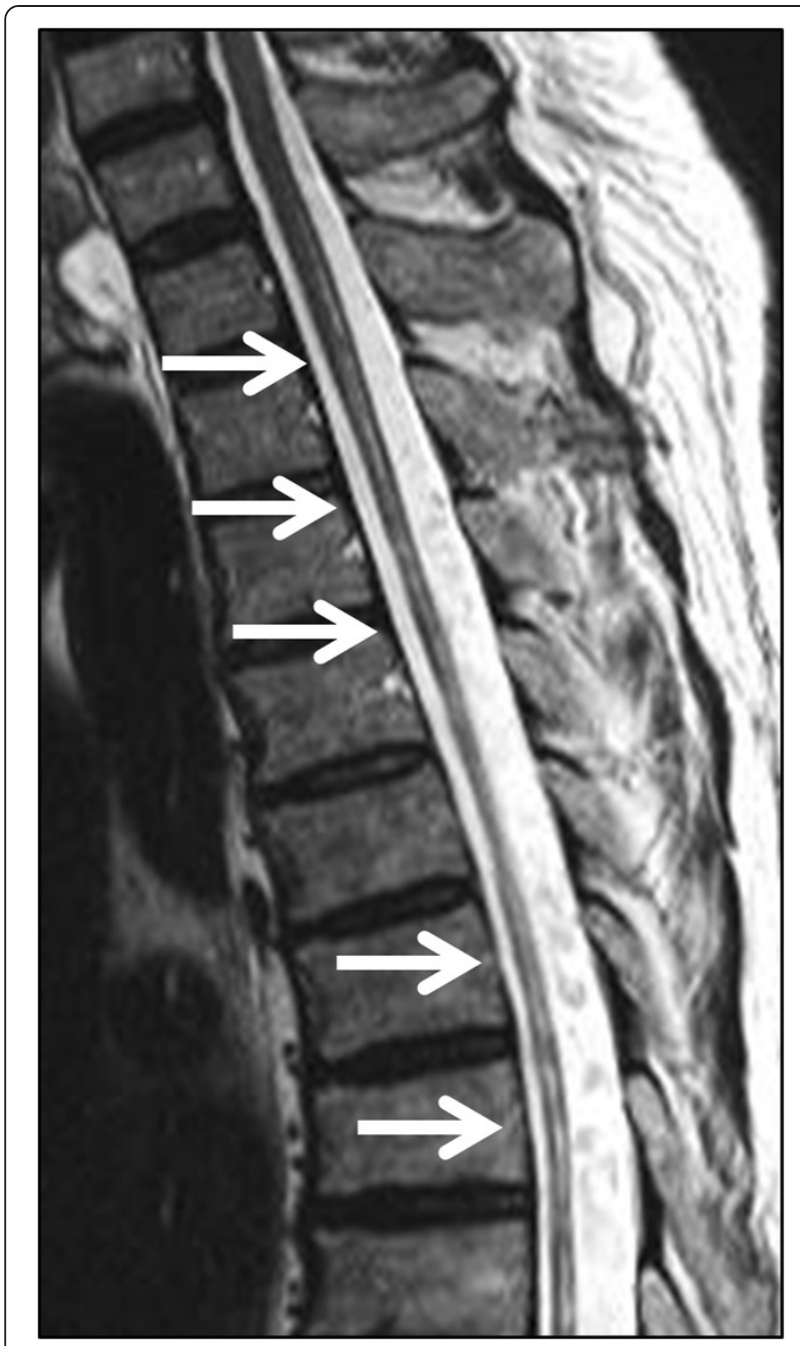

Figure 1 Spinal cord magnetic resonance imaging (T2-weighted imaging from (4 to Th6) one year after the start of combination treatment with rituxan and cyclophosphamide depicting longitudinally extensive spinal cord lesions (see arrows) with significant cord atrophy.

criteria. Moreover, analysis of the cerebrospinal fluid (CSF) presented oligoclonal bands. At that time, anti-AQP-4 antibody testing was not performed. A therapy with interferon beta 1a was started for six months and was replaced by interferon beta $1 b$ at the discretion of the treating outside neurologist. Our patient developed two further spinal relapses during the treatment with interferon beta preparations. They were treated with corticosteroid pulses without any success and his EDSS score worsened from 2.5 to 4.0. Although a subsequent therapy with natalizumab was initiated at an outside clinic, our patient continued to present another three relapses. The first relapse occurred four months after starting natalizumab, the second after six months and the third relapse after eight months. All relapses repeatedly affected both optic nerves and the spinal cord each with increasing visual and motor impairment.
Thus, our patient developed a high-grade spastic tetraparesis as well as impaired visual acuity of both eyes and his EDSS score progressed from 4.0 to 8.0. At that point, NMO was discussed after referral to our hospital and natalizumab therapy was discontinued after nine courses. After repeated cycles of plasma exchange, the disease course stabilized and a therapy with rituxan was started. Although B cells were completely depleted, our patient experienced another severe myelitis relapse upon further follow-up three months later. Consequently, an additional immunosuppressive therapy with cyclophosphamide at a dosage of 600mg/ $\mathrm{m}^{2}$ was initiated. In the meantime, we performed repetitive anti-AQP-4 antibody tests in an approved external laboratory employing an immunofluorescence assay (IFA) cellbased analysis. Negative anti-AQP-4 antibody tests were obtained via IFA analyses upon first admission and then in six-monthly intervals after first contact at our hospital. It was only after 18 months that anti-AQP-4 antibodies became positive after three negative results. At that time, the anti-AQP-4-immunoglobulin (Ig)G antibody titer was 1:1000 while IgM and IgA titers were negative. There were no other autoantibodies and no signs of other autoimmune diseases or malignancy.

Under combination treatment with cyclophosphamide (13 cycles every six weeks, cumulative dosage of $8300 \mathrm{mg} / \mathrm{m}^{2}$ ) followed by another cycle of rituxan, our patient developed no further relapses over an observation period of 2.5 years.

\section{Conclusions}

In this case NMO was initially misdiagnosed as MS, whereas CSF analysis revealed positive oligoclonal bands and an MRI scan of the brain presented some periventricular lesions without fulfilling the Barkhof criteria. However, oligoclonal bands may be positive in up to 30 percent of NMO patients and 60 percent of the patients may present supratentorial lesions [8]. In our patient, no follow-up CSF analysis was available to check for the persistence of oligoclonal bands.

After first- and second-line immunomodulatory therapy failed, natalizumab was chosen as an escalation therapy at an outside hospital. In at least one case series and two further case reports, a total of six NMO patients presented continuous relapses or even a deteriorating disease course under natalizumab, [6,9-11]. Subsequently, these observations were controversially discussed in the neuroimmunological community [12]. Our case adds further evidence to the notion that natalizumab, while highly effective in MS, may not work sufficiently in NMO. Indeed, natalizumab does not positively affect the trafficking properties of neutrophils, which are abundant in NMO lesions. After natalizumab therapy, an increase of B-cell precursors in the blood is observed, which might be counterproductive in case of a presumed B-cell-based pathomechanism as in NMO $[13,14]$. 
Our patient only stabilized after treatment with rituxan combined with cyclophosphamide. While cyclophosphamide alone may not work sufficiently in NMO [15], a combination treatment regime of complete B-cell depletion followed by cyclophosphamide pulses similar to rheumatologic disease may be more effective, at least in the present case. Nowadays, treatment with tocilizumab may be another option in rituxan-refractory NMO [7].

In our case, a first positive test for anti-AQP-4 antibodies occurred eight years after the onset of his first symptoms during a severe relapse. It is interesting to discuss whether this finding represents true seroconversion, is linked to variable test sensitivities or is governed by testing during a relapse. Indeed, titers of anti-AQP-4 antibodies might reflect the disease activity of NMO patients [16]. Thus, repeated testing in the active phases of the disease may prove useful. We thus propose critical testing for $\mathrm{NMO}$ in all patients with poorly controlled autoimmune central nervous system (CNS) inflammation, especially before, but also after initiation of natalizumab therapy. If NMO is clinically suspected but initial anti-AQP-4 antibody testing remains negative, repeated tests may be helpful to confirm the final diagnosis.

\section{Consent}

Written informed consent was obtained from the patient for publication of this case report and any accompanying images. A copy of the written consent is available for review by the Editor-in-Chief of this journal.

\section{Abbreviations \\ AQP-4: aquaporin-4; CNS: central nervous system; CSF: cerebrospinal fluid; EDSS: expanded disability status scale; IFA: immunofluorescence assay; Ig: immunoglobulin; MRI: magnetic resonance imaging; MS: multiple sclerosis; NMO: neuromyelitis optica.}

\section{Competing interests}

The authors declare that they have no competing interests.

\section{Authors' contributions}

$\mathrm{DL}, \mathrm{AL}$ and $\mathrm{RL}$ took the lead in drafting the manuscript and have made substantial contributions to interpretation of data. CM and SS have been involved in drafting the manuscript. AW and TS provided help with magnetic resonance imaging and critically revising the text. RL and CM revised it critically for important intellectual content. RL has given final approval of the version to be published. All authors read and approved the final manuscript.

\section{Author details \\ 'Department of Neurology, Friedrich-Alexander University of Erlangen-Nuremberg, Schwabachanlage 6, 91054 Erlangen, Germany. ${ }^{2}$ Department of Neuroradiology, Friedrich-Alexander University of Erlangen-Nuremberg, Schwabachanlage 6, 91054 Erlangen, Germany.}

Received: 30 December 2013 Accepted: 17 March 2014

Published: 19 May 2014

\section{References}

1. Wingerchuk DM, Lennon VA, Pittock SJ, Lucchinetti CF, Weinshenker BG: Revised diagnostic criteria for neuromyelitis optica. Neurology 2006, 66:1485-1489.

2. Lennon VA, Kryzer TJ, Pittock SJ, Verkman AS, Hinson SR: IgG marker of optic- spinal multiple sclerosis binds to the aquaporin-4 water channel. J Exp Med 2005, 204:473-477.
3. Papeix C, Vidal JS, de Seze J, Pierrot-Deseilligny C, Tourbah A, Stankoff B, Lebrun C, Moreau T, Vermersch P, Fontaine B, Lyon-Caen O, Gout O: Immunosuppressive therapy is more effective than interferon in neuromyelitis optica. Mult Scler 2007, 13:256-259.

4. Trebst C, Jarius S, Berthele A, Paul F, Schippling S, Wildemann B, Borisow N, Kleiter I, Aktas O, Kümpfel T, Neuromyelitis Optica Study Group (NEMOS): Update on the diagnosis and treatment of neuromyelitis optica: recommendations of the Neuromyelitis Optica Study Group (NEMOS). J Neurol 2014, 261:1-16.

5. Jacob A, Weinshenker BG, Violich I, McLinskey N, Krupp L, Fox RJ, Wingerchuk DM, Boggild M, Constantinescu CS, Miller A, De Angelis T, Matiello M, Cree BA: Treatment of neuromyelitis optica with rituximab: retrospective analysis of 25 patients. Arch Neurol 2008, 65:1443-1448.

6. Barnett MH, Prineas JW, Buckland ME, Parratt JD, Pollard JD: Massive astrocyte destruction in neuromyelitis optica despite natalizumab therapy. Mult Scler 2012, 18:108-112.

7. Ayzenberg I, Kleiter I, Schröder A, Hellwig K, Chan A, Yamamura T, Gold R: Interleukin 6 receptor blockade in patients with neuromyelitis optica nonresponsive to anti-CD20 therapy. JAMA Neurol 2013, 70:394-397.

8. Jarius S, Ruprecht K, Wildemann B, Kuempfel T, Ringelstein M, Geis C, Kleiter I, Kleinschnitz C, Berthele A, Brettschneider J, Hellwig K, Hemmer B, Linker RA, Lauda F, Mayer CA, Tumani H, Melms A, Trebst C, Stangel M, Marziniak M, Hoffmann F, Schippling S, Faiss JH, Neuhaus O, Ettrich B, Zentner C, Guthke K, Hofstadt-van Oy U, Reuss R, Pellkofer $\mathrm{H}$, et al: Contrasting disease patterns in seropositive and seronegative neuromyelitis optica: a multicentre study of 175 patients. J Neuroinflammation 2012, 19:14.

9. Kleiter I, Hellwig K, Berthele A, Kümpfel T, Linker RA, Hartung HP, Paul F, Aktas O, Neuromyelitis Optica Study Group: Failure of natalizumab to prevent relapses in neuromyelitis optica. Arch Neurol 2012, 69:239-245.

10. Jacob A, Hutchinson M, Elsone L, Kelly S, Ali R, Saukans I, Tubridy N, Boggild M: Does natalizumab therapy worsen neuromyelitis optica? Neurology 2012, 79:1065-1066.

11. Juryńczyk M, Zaleski K, Selmaj K: Natalizumab and the development of extensive brain lesions in neuromyelitis optica. J Neurol 2013, 260:1919-1921.

12. Govindarajan $\mathrm{R}$, Salgado E: Is it too early to predict the failure of natalizumab in NMO? Arch Neurol 2012, 69:1085.

13. Kim SH, Kim W, Li XF, Jung IJ, Kim HJ: Repeated treatment with rituximab based on the assessment of peripheral circulating memory B cells in patients with relapsing neuromyelitis optica over 2 years. Arch Neurol 2011, 68:1412-1420.

14. Krumbholz M, Meinl I, Kümpfel T, Hohlfeld R, Meinl E: Natalizumab disproportionately increases circulating pre-B and $B$ cells in multiple sclerosis. Neurology 2008, 71:1350-1354.

15. Bichuetti DB, Oliveira EM, Boulos Fde C, Gabbai AA: Lack of response to pulse cyclophosphamide in neuromyelitis optica: evaluation of 7 patients. Arch Neurol 2012, 69:938-939.

16. Weinshenker BG, Wingerchuk DM, Vukusic S, Linbo L, Pittock SJ, Lucchinetti CF, Lennon VA: Neuromyelitis optica IgG predicts relapse after longitudinally extensive transverse myelitis. Ann Neurol 2006, 59:566-569.

doi:10.1186/1752-1947-8-155

Cite this article as: Lee et al:: Neuromyelitis optica presenting with relapses under treatment with natalizumab: a case report. Journal of Medical Case Reports 2014 8:155.

\section{Submit your next manuscript to BioMed Central and take full advantage of:}

- Convenient online submission

- Thorough peer review

- No space constraints or color figure charges

- Immediate publication on acceptance

- Inclusion in PubMed, CAS, Scopus and Google Scholar

- Research which is freely available for redistribution 